\title{
THE EFFICIENCY OF AN ACTIVE GENERATOR IN THE CASE OF A DEEP FOUNDATION LOCATION
}

\author{
ANETA HERBUt \\ Wrocław University of Science and Technology, Faculty of Civil Engineering, \\ Wybrzeże Wyspiańskiego 27, 50-370 Wrocław, Poland, e-mail: aneta.herbut@pwr.edu.pl
}

\begin{abstract}
This paper presents a concept for vibration-mitigation techniques with the potential to reduce ground vibration amplitudes by applying an additional vibration source. The idea of an additional generator is verified in the case of an impact load for the points located on the ground surface and below it. Equations of motion for the damped transversally isotropic ground model with the absorbing boundary conditions are presented and numerically integrated using FlexPDE software, based on the finite element method. The efficiency of the solution is analyzed in terms of reducing the vertical and horizontal components. Results are presented in the form of a dimensionless amplitude reduction factor. In each case being analyzed, a vibration mitigation effect in a three-story building was achieved.
\end{abstract}

Key words: vibration-mitigation techniques, wave propagation, soil dynamics

\section{INTRODUCTION, LITERATURE REVIEW}

In this paper, an original approach for protecting structures against large vibration amplitudes resulting from ground-applied loads is presented. The idea is to apply an additional vibration source and to generate new vibrations with similar vibration amplitudes and frequencies but directed opposite to the ones being attenuated. Summing the displacements of these two sources will significantly decrease the vibration. The idea of an additional generator has already been presented in the case of harmonic excitation (Herbut [11]). The efficiency of this solution was verified by different soil conditions and locations of an additional generator. The results were presented in the form of dimensionless amplitude reduction factor for the points located on the ground surface (Herbut [11]). In the present paper, the idea is examined by a different type of excitation - by the case of an impact load. The results are presented not only for points located on the ground surface, but also the ones deeper in the ground. According to the different European Standards (DIN 4150-3, 1999 [9]; BS 5228-4, 1992 [2]; BS 7385-2, 1993 [3]) vibration should be monitored both on the foundation level and on the structure, in order to prevent vibration amplifications due to resonance effect. That is why the efficiency of additional generators for a three-story building is also verified. However, the main concept of the present paper is to examine if the idea of an additional generator can be still effective for structures with underground floors, with foundations located deeper below the ground surface or underground structures.

Two different approaches are used in structural control to prevent large vibration amplitudes. By the first one the dissipative characteristics of the structure are increased or natural frequencies of structure are moved away from the predicted excitation frequencies. Passive, active or semi-active systems are applied to the structure (Chu et al. [6]). By the second approach the amplitudes of ground vibration are decreased before the wave reaches the structure.

Passive control does not require an external power source. This approach includes passive isolations, viscoelastic dampers or tuned mass dampers. Because passive systems are unable to adapt to changing loading conditions, damping forces are not always optimized. This is in contrast to active and semi-active systems, which are able to change their damping characteristics for a better reduction effect (Pakos et al. [16]). However, these systems require an external power source. The energy requirements for semiactive systems are smaller than those for active systems (Chu et al. [6]). The idea described above is addressed to new buildings/structures. Applying the control devices to existing structures is too invasive and costly. However, it is possible to modify the energy transferred to the structure. The concept of 
vibration screening provides the possibility to control the energy reaching the sensitive zones. The most common method to decrease the amplitudes of ground motion is the use of the wave barrier in the form of vertical or horizontal obstacles. A barrier in the form of a ground discontinuity eliminates the possibility of wave propagation. A vibration attenuation effect is achieved by the proper interception, scattering and diffraction of surface waves. Different solutions are implemented to achieve wave scattering, including sheetpile walls (Dijckmans et al. [8]), piles (Kattis et al. [12], Gao et al. [10], Turan et al. [19]) and in-filled trenches - with water, soft soil, soil-bentonite mixture, concrete or special materials, such as GeoFoam (Ulgen and Toygar [20], Alzawi et al. [1], Coulier et al. [7]). However, the best results are achieved by the use of so called open trenches (Kattis et al. [12], Leung et al. [13]). Apart from separated barrier or rows of obstacles in the ground, shaping landscapes can give satisfactory results in Rayleigh wave attenuation (Persson et al. [17]).

The present paper describes a concept for vibration-mitigation techniques with the potential to reduce the amplitudes of ground vibration by applying an additional vibration source. From the point of view of energy requirements, the proposed solution is similar to active systems applied to structures, but it does not interfere with the structure. It attenuates a vibration amplitude before a wave reaches a structure. That makes the solution similar to ground barriers. However, the approach is easier, less expensive and faster to implement than the two aforementioned methods. It is also portable and can be used repeatedly in different places and situations. Additional generator is addressed to man-made vibrations, induced by machines, dynamic compaction, thus to the case when the Rayleigh wave is dominant.

\section{MATHEMATICAL MODEL}

\subsection{EQUATIONS OF MOTION FOR A DAMPED TRANSVERSALLY ISOTROPIC MEDIUM}

To assume a correct model for a soil medium, the strains must be estimated. For a relatively large strain (shear strain exceeding $10^{-4}$ ) a two-phase elasticplastic soil medium model based on the Biot theorem is necessary (Wrana [23]). In the example presented, the problem is analyzed in the range of small deformations, so the elastic one-phase model is assumed.
This model is typically assumed in similar investigations considering wave propagation in soil and vibration mitigation techniques (Coulier et al. [7], Chen et al. [5], Turan et al. [19], Gao et al. [10], Kattis et al. [12]).

Wave propagation phenomenon in a transversally isotropic soil medium with hysteretic damping can be described by the following equations (Verruijt [21])

$$
\begin{aligned}
\Sigma P_{x} & =0: \frac{\partial \sigma_{x}}{\partial x}+\frac{\partial \tau_{y x}}{\partial y}+\frac{\partial \tau_{z x}}{\partial z}+t r \cdot \frac{\partial \sigma_{x}}{\partial t \partial x} \\
& +t r \cdot \frac{\partial \tau_{y x}}{\partial t \partial y}+t r \cdot \frac{\partial \tau_{z x}}{\partial t \partial z}=\rho \frac{\partial^{2} u}{\partial t^{2}}, \\
\Sigma P_{z} & =0: \frac{\partial \tau_{x y}}{\partial x}+\frac{\partial \sigma_{y}}{\partial y}+\frac{\partial \tau_{z y}}{\partial z}+t r \cdot \frac{\partial \tau_{x y}}{\partial t \partial x} \\
& +\operatorname{tr} \cdot \frac{\partial \sigma_{y}}{\partial t \partial y}+t r \cdot \frac{\partial \tau_{z y}}{\partial t \partial z}=\rho \frac{\partial^{2} v}{\partial t^{2}}, \\
\Sigma P_{y} & =0: \frac{\partial \tau_{x z}}{\partial x}+\frac{\partial \tau_{y z}}{\partial z}+\frac{\partial \sigma_{z}}{\partial y}+t r \cdot \frac{\partial \tau_{x z}}{\partial t \partial x} \\
& +t r \cdot \frac{\partial \tau_{y z}}{\partial t \partial y}+t r \cdot \frac{\partial \sigma_{z}}{\partial t \partial z}=\rho \frac{\partial^{2} w}{\partial t^{2}},
\end{aligned}
$$

where $u, v$ and $w$ are the components of displacement in the $x$-, $y$ - and $z$-directions, respectively; $\rho$ is the soil density; $\sigma_{x}, \sigma_{y}, \sigma_{z}, \tau_{x y}=\tau_{y x}, \tau_{x z}=\tau_{z x}, \tau_{y z}=\tau_{z y}$ are normal and shear elastic stresses, respectively; and $t r$ is the relaxation time, which is inversely proportional to the excitation frequency $t r=\frac{2 \xi}{\omega}$, where $\xi$ is the damping coefficient. The relations for strains in terms of displacements are assumed as: $\varepsilon_{x}=\frac{\partial u}{\partial x}$, $\varepsilon_{y}=\frac{\partial v}{\partial y}, \quad \varepsilon_{z}=\frac{\partial w}{\partial z}, \quad \gamma_{x y}=\frac{\partial v}{\partial x}+\frac{\partial u}{\partial y}, \quad \gamma_{x z}=\frac{\partial w}{\partial x}+\frac{\partial u}{\partial z}$, $\gamma_{y z}=\frac{\partial w}{\partial y}+\frac{\partial v}{\partial z}$. For an elastic transversally isotropic material with a horizontal plane of isotropy, the elastic strain stress relationship can be presented as follows (Carcione [4])

$$
\left[\begin{array}{c}
\varepsilon_{x} \\
\varepsilon_{y} \\
\varepsilon_{z} \\
\gamma_{y z} \\
\gamma_{x z} \\
\gamma_{x y}
\end{array}\right]=\left[\begin{array}{cccccc}
C_{11} & C_{12} & C_{13} & 0 & 0 & 0 \\
C_{12} & C_{11} & C_{12} & 0 & 0 & 0 \\
C_{13} & C_{13} & C_{33} & 0 & 0 & 0 \\
0 & 0 & 0 & C_{55} & 0 & 0 \\
0 & 0 & 0 & 0 & C_{55} & 0 \\
0 & 0 & 0 & 0 & 0 & C_{66}
\end{array}\right]\left[\begin{array}{c}
\sigma_{x} \\
\sigma_{y} \\
\sigma_{z} \\
\tau_{y z} \\
\tau_{x z} \\
\tau_{x y}
\end{array}\right],
$$


where $C_{11}=\frac{1}{E_{x}}, C_{12}=-\frac{v_{x}}{E_{x}}, C_{13}=-\frac{v_{z}}{E_{z}}, C_{33}=\frac{1}{E_{z}}$, $C_{55}=\frac{1}{G_{x z}}$ and $C_{66}=\frac{2\left(1+v_{x}\right)}{E_{x}} . E_{x}, E_{y}=E_{x}$ are Young's moduli in the $x$ - and $y$-directions, respectively, in the plane of isotropy. $E_{z}$ is Young's modulus in the $z$-direction, in the plane perpendicular to the plane of isotropy. Similarly $v_{x}, v_{y}=v_{x}$ and $v_{z}$ are Poisson's ratios in the plane of isotropy and in the plane perpendicular to the plane of isotropy, respectively. $G_{x z}$ is the shear modulus in the plane perpendicular to the plane of isotropy.

\subsection{BOUNDARY CONDITIONS AND FINITE ELEMENT MODEL}

The absorbing viscous boundary conditions are assumed following Lysmer and Kuhlemeyer [14]. They are applied to the model to avoid wave reflection at the boundary. The normal $\left(\sigma_{x}\right)$ and shear $\left(\tau_{x y}, \tau_{x z}\right)$ stress components for virtual dampers "fixed" to the right (along coordinate $x=70 \mathrm{~m}$ in Fig. 1) and left (along coordinate $x=0$ in Fig. 1) boundaries are given by the formulas

$$
\sigma_{x}=a \rho V_{x} \dot{u}, \quad \tau_{x y}=b \rho V_{x y} \dot{v}, \quad \tau_{x z}=b \rho V_{x z} \dot{w},
$$

where $\rho$ is the mass density, $\dot{u}, \dot{v}$ and $\dot{w}$ are velocities in the $x$-, $y$ - and $z$-directions, respectively, $a$ and $b$ are parameters introduced to improve the wave absorption at the boundaries in the normal and tangential directions, respectively. Research findings indicate that $a=1$ and $b=0.25$ can yield reasonable absorption at the boundary (Turan et al. [19]). These values are also assumed in this study. $V_{x}$ denotes the $\mathrm{P}$-wave velocity, and $V_{x y}, V_{x z}$ denote the S-wave velocities. The notation $V_{i j}$ indicates propagation in the $i$-direction, whereas the polarization is in the $j$-direction. The explanation of the wave propagation phenomenon by different types of anisotropy including determination of wave velocities is omitted here, as it is widely discussed in the literature (Carcione [4]). The velocities in the plane of isotropy of the transversally isotropic medium are defined as $V_{x}=V_{y}=\sqrt{\frac{C_{11}}{\rho}}$, $V_{y z}=\sqrt{\frac{C_{55}}{\rho}}, V_{x z}=\sqrt{\frac{C_{55}}{\rho}}$ and $V_{x y}=V_{y x}=\sqrt{\frac{C_{66}}{\rho}}$ (Carcione [4]). In the plane perpendicular to the plane of isotropy the velocities can be calculated from the following formulas: $V_{z}=\sqrt{\frac{C_{33}}{\rho}}, V_{z y}=\sqrt{\frac{C_{55}}{\rho}}$, and $V_{z x}=\sqrt{\frac{C_{55}}{\rho}}$.
The normal $\left(\sigma_{y}\right)$ and shear $\left(\tau_{y x}, \tau_{y z}\right)$ stress components in the virtual dampers "fixed" to the near and far edges of the analyzed domain (along coordinates $y=0$ and $y=70 \mathrm{~m}$, see Fig. 1) are expressed as

$$
\sigma_{y}=a \rho V_{y} \dot{v}, \quad \tau_{y x}=b \rho V_{y x} \dot{u}, \quad \tau_{y z}=b \rho V_{y z} \dot{w} .
$$

For the bottom boundary $(z=-35 \mathrm{~m})$ damping forces are described by the formulas

$$
\sigma_{z}=a \rho V_{z} \dot{w}, \quad \tau_{z x}=b \rho V_{z x} \dot{u}, \quad \tau_{z y}=b \rho V_{z y} \dot{v} .
$$

Additionally, both displacement components are assumed to be zero at the bottom edge of the region investigated.

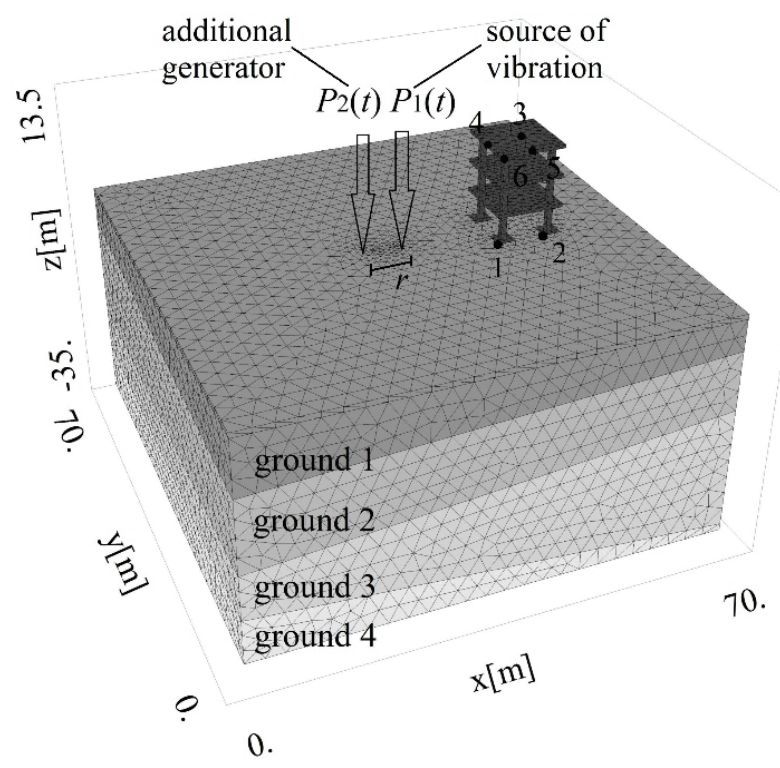

Fig. 1. Domain with the vibration source and additional generator

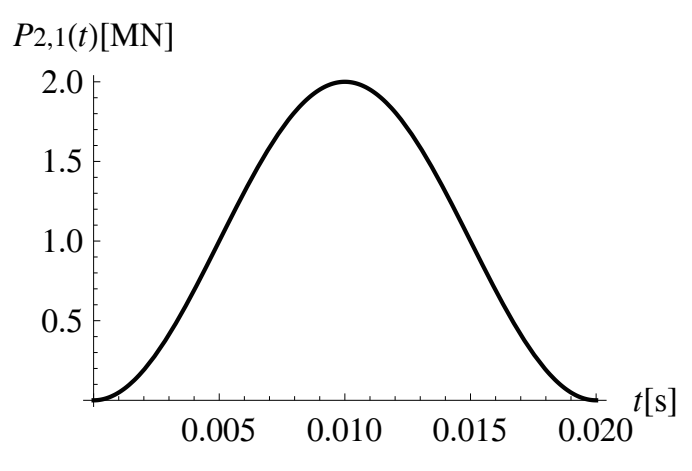

Fig. 2. Force generated by an additional generator, the first impact, haversine function

A haversine load of amplitude $2 \mathrm{MN}$ and duration $d=0.02 \mathrm{~s}$ is applied to the ground surface, as in Figs. 1 and 2 (Mabsout and Tassoulas [15]). The period of a hammer blow is $T=1 \mathrm{~s}$. 
Each separated $i$-th hammer blow is described by haversine function $P_{1, i}(t)$

$$
\begin{gathered}
P_{1, i}(t)=0.5 A\left(1-\cos \left(\frac{2 \pi\left(t-t_{b, i}\right)}{t_{e, i}-t_{b, i}}\right)\right) \\
\cdot\left(\mathrm{H}\left(t-t_{b, i}\right)-\mathrm{H}\left(t-t_{e, i}\right)\right) \\
P_{1, i}(t)=\sum_{i=1}^{n} P_{1, i}(t)
\end{gathered}
$$

where $A$ is the amplitude of the excitation, $\mathrm{H}(t)$ is the Heaviside function, $t_{b, i}$ is the time when the impact begins, $t_{e, i}$ is the time when it finishes. For the ana-

lyzed example, $t_{b, i}=(i-1) \cdot T+\frac{r}{V_{\mathrm{R}, 1}}, t_{e, i}=t_{b, i}+d$,

where $V_{R, 1}$ is the Rayleigh wave velocity for the first layer, $n$ is the number of blows considered. The force is located in the middle of the region considered ( $x=35 \mathrm{~m}, y=35 \mathrm{~m}, z=0)$ and applied to a square concrete element with a width of $0.4 \mathrm{~m}$. Force $P_{1}(t)$ is similar to $P_{2}(t)$ in Fig. 2 but "moved" along the $t$-axis. The additional generator $P_{2}(t)$ is used to mitigate the vibration amplitudes generated by $P_{1}(t)$ (Figs. 1, 2). It is located at a distance $r$ on the left side of the applied load $(x=35 \mathrm{~m}-r$, $y=35 \mathrm{~m}, z=0)$. The force generated by the additional generator can be expressed as

$$
\begin{gathered}
P_{2, i}(t)=-0.5 A\left(1-\cos \left(\frac{2 \pi\left(t-t_{B, i}\right)}{t_{E, i}-t_{B, i}}\right)\right) \\
\cdot\left(\mathrm{H}\left(t-t_{B, i}\right)-\mathrm{H}\left(t-t_{E, i}\right)\right), \\
P_{2, i}(t)=\sum_{i=1}^{n} P_{2, i}(t),
\end{gathered}
$$

where $t_{B, i}$ is the time when the impact of the additional generator starts, and $t_{E, i}$ is the time when it finishes. For the analyzed example, $t_{B, i}=(i-1)$ - $T, t_{E, i}=t_{B, i}+d$. The time delay between two exciters $\mathrm{P}_{1}(t)$ and $\mathrm{P}_{2}(t)$ is introduced to improve the efficiency of the system. The additional generator starts working earlier. The shift in time corresponds to the time needed for the Rayleigh wave to cover the distance $r$.

The presented partial differential equations with the appropriate absorbing boundary conditions are solved using FlexPDE Professional V6 software based on the finite element method. Four-noded linear tetrahedron finite elements with three degrees of freedom at each node are assumed. A fully bonded soil foundation interface is assumed.

\section{RESULTS AND CONCLUSIONS}

To compare the results obtained before and after using the additional generator the non-dimensional factor AMF is introduced after Woods [22]. The amplitude mitigation factor (AMF) for the vertical displacement component is expressed as

$$
A M F_{z}=\operatorname{Max}(\operatorname{Abs}(w)) / \operatorname{Max}\left(\operatorname{Abs}\left(w_{0}\right)\right) .
$$

The calculations are performed for two cases: with $(w)$ and without $\left(w_{0}\right)$ the use of an additional generator. For both situations, the maximum absolute values of the displacement components at each of the 200 points are established, and the AMF is then evaluated. The analyses are made for all three displacement components. The displacements are considered for the time interval $3.5 \mathrm{~s}$. The appropriate partial differential equations are solved using FlexPDE Professional V6 software. Then the obtained results are analyzed using Mathematica 7.

The aim of this section is to verify the efficiency of an additional generator for points located deeper below the ground surface - to the depth of $10 \mathrm{~m}$. This issue can be important for structures with underground floors or underground structures. Two different soil conditions are examined. In the first case, the dynamical properties of the shallow located layer are better $G_{x z, 1}=2 G_{x z, 2}$ (Section 3.1). In the second case, the situation is opposite - the second layer's dynamical properties are better $G_{x z, 2}=2 G_{x z, 1}$ (Section 3.2). The results are presented in the form of AMF for all three displacement components, for the points located on the ground surface, at a the depth of $5 \mathrm{~m}$ and in the middle of the region considered to the depth of $10 \mathrm{~m}$ (cross-section along $y=35 \mathrm{~m}$ ).

\subsection{THE FIRST LAYER'S DYNAMICAL PROPERTIES ARE BETTER}

The transversally isotropic, layered half space is assumed (Fig. 1). For the shallow deposit, the dynamic soil parameters are as follows: shear modulus $G_{x z, 1}=160 \mathrm{MPa}$; Young's modulus in the plane of isotropy $E_{x, 1}=3.865 G_{x z, 1}$; Young's modulus in the plane perpendicular to the plane of isotropy $E_{z, 1}=$ $2.863 G_{x z, 1}$; Poisson's ratios: $v_{x, 1}=0.280$ and $v_{z, 1}=$ 0.165 ; mass density of the soil $\rho_{1}=2000 \mathrm{~kg} / \mathrm{m}^{3}$ and damping coefficient $\xi=1 \%$. The boundary surface between the first and second layers is inclined in both directions $(x$ and $y)$. It is defined by the following points: $P_{1}(0,0,-10 \mathrm{~m}), P_{2}(70 \mathrm{~m}, 0,-5 \mathrm{~m})$ and $P_{3}(70 \mathrm{~m}, 70 \mathrm{~m}$, 
$-2 \mathrm{~m})$. For the second layer, the dynamic soil parameters are as follows: shear modulus $G_{x z, 2}=$ $80 \mathrm{MPa}$; Young's moduli: $E_{x, 2}=3.865 G_{x z, 2}$ and $E_{y, 2}=$ $2.863 G_{x z, 2}$; Poisson's ratios: $v_{x, 2}=0.301$ and $v_{z, 2}=$ 0.182 ; damping coefficient $\xi=1 \%$; and mass density of the soil $\rho_{2}=2000 \mathrm{~kg} / \mathrm{m}^{3}$ (Chen et al. 2014). The boundary surface between the second and third layers is inclined but only in one direction. It is defined by the following points: $P_{4}(0,0,-21 \mathrm{~m}), P_{5}(70 \mathrm{~m}, 0$, $-15 \mathrm{~m})$ and $P_{6}(70 \mathrm{~m}, 70 \mathrm{~m},-15 \mathrm{~m})$. For the third layer, the dynamic soil parameters are as follows: shear modulus $G_{x z, 3}=560 \mathrm{MPa}$; Young's moduli: $E_{x, 3}=3.865 G_{x z, 3}$ and $E_{y, 3}=2.863 G_{x z, 3}$; Poisson's ratios: $v_{x, 3}=0.250$ and $v_{z, 3}=0.160$; damping coefficient $\xi=1 \%$; and mass density of the soil $\rho_{3}=2000 \mathrm{~kg} / \mathrm{m}^{3}$. The boundary surface between the third and fourth layers is defined by the following points: $P_{7}(0,0$,
$-28 \mathrm{~m}), P_{8}(70 \mathrm{~m}, 0,-34 \mathrm{~m})$ and $P_{9}(70 \mathrm{~m}, 70 \mathrm{~m}$, $-34 \mathrm{~m})$. For the fourth layer, the dynamic soil parameters are as follows: shear modulus $G_{x z, 4}=800 \mathrm{MPa}$; Young's moduli: $E_{x, 4}=3.965 G_{x z, 4}$ and $E_{y, 4}=$ $2.863 G_{3, x z}$; Poisson's ratios: $v_{x, 4}=0.230$ and $v_{z, 4}=$ 0.150 ; damping coefficient $\xi=1 \%$; and mass density of the soil $\rho_{4}=2000 \mathrm{~kg} / \mathrm{m}^{3}$. For the concrete elements, the isotropic material model is assumed with dynamic parameters: $G_{x z}=12.2 \mathrm{GPa}, E_{x}=E_{y}=27 \mathrm{GPa}, v_{x}=v_{z}$ $=0.176, \rho=2500 \mathrm{~kg} / \mathrm{m}^{3}, \xi=1 \%$. The three-story building is located on the right side of the analyzed domain at a distance of $20 \mathrm{~m}$ from the vibration source $P_{1}(t)$ (Fig. 1). The square plates in each story are $10 \mathrm{~m}$ wide and $0.5 \mathrm{~m}$ thick. Each story is $4 \mathrm{~m}$ high. The concrete square columns ( $0.8 \mathrm{~m}$ wide) are coupled with the concrete square foundation footings, each of which is $3 \mathrm{~m}$ wide and $0.8 \mathrm{~m}$ thick.
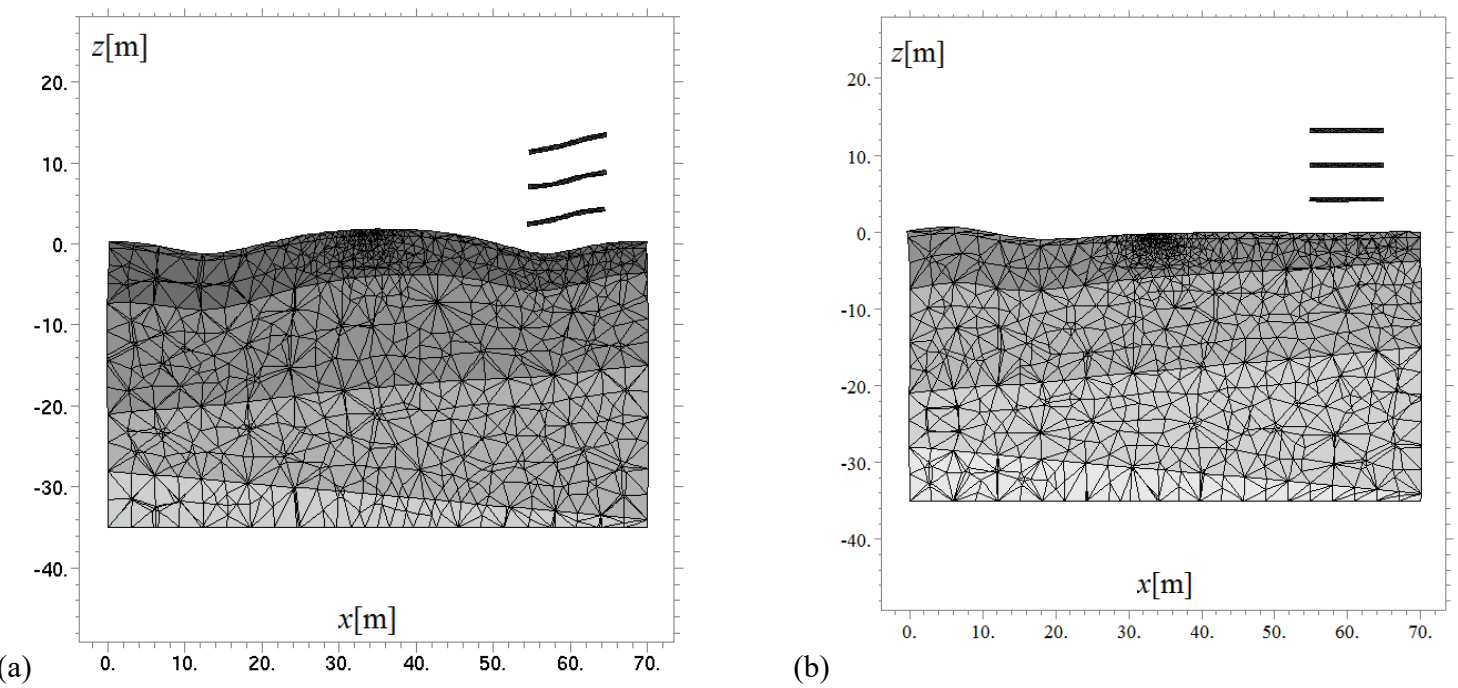

Fig. 3. Ground and structure deformation for $y=35 \mathrm{~m}, t=0.125 \mathrm{~s}$;

(a) additional generator is removed, (b) additional generator is used
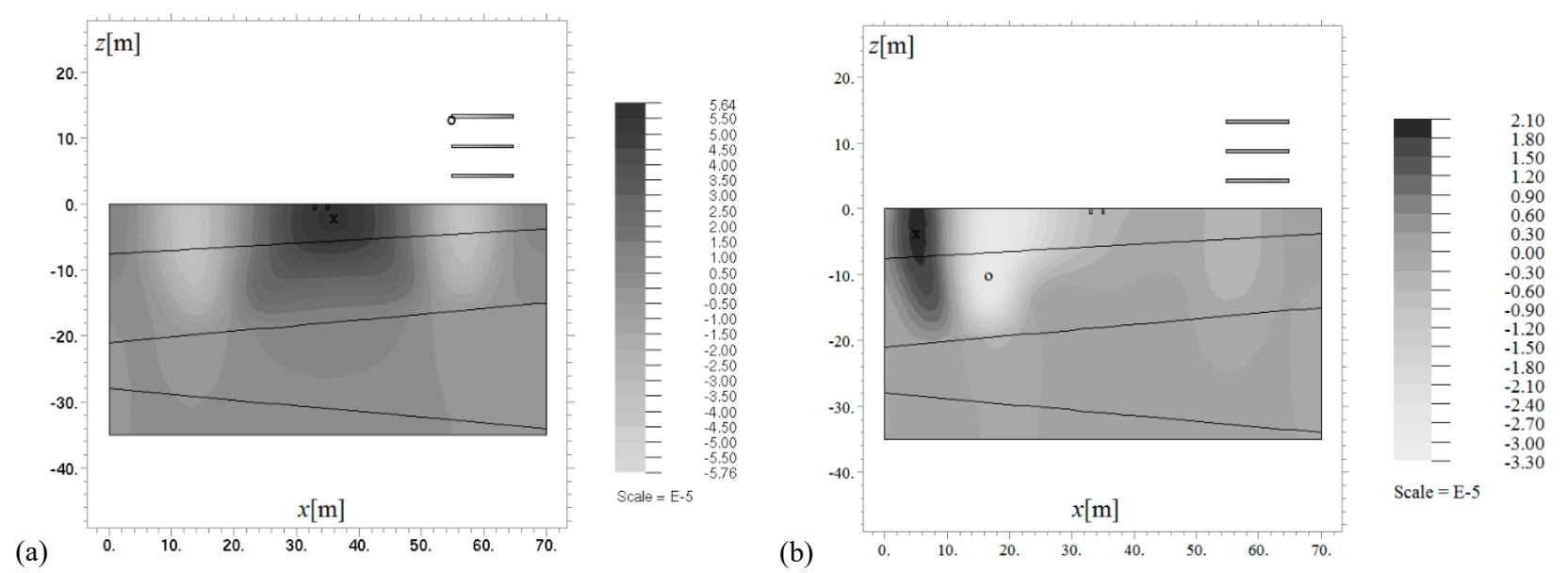

Fig. 4. Displacement component in $z$-direction in [m] for $y=35 \mathrm{~m}, t=0.125 \mathrm{~s}$; (a) additional generator is removed, (b) additional generator is used 
The question is whether the idea of using an additional source of vibration in order to reduce the large vibration amplitudes can be effective? The deformation of the ground surface and the structure is presented before (Fig. 3a) and after using the additional generator (Fig. 3b), for $t=0.125 \mathrm{~s}$. The results are presented in the middle of the analyzed region along the cross-section $y=35 \mathrm{~m}$ (Fig. 1). The significant reduction effect can be obtained for both - the ground surface and the structure. In Fig. 4, the vertical displacement component is presented before (Fig. 4a) and after using the additional generator (Fig. 4b), for the same point in time $t=0.125 \mathrm{~s}$. It can be observed that the mitigation effect is achieved for the points located on the right side of the region considered ( $x$ from $35 \mathrm{~m}$ to $70 \mathrm{~m}$ ), not only on the ground surface, but also deeper below it (Fig. 4a, Fig. 4b).

For a better comparison the non-dimensional parameter AMF is introduced after Woods (8). The additional generator's efficiency in the form of AMF for all displacement components, for the points located on the ground surface is presented in Fig. 5a-c. For example, $\mathrm{AMF}=0.4$ means that a $60 \%$ reduction was achieved. The mitigation of vibration can be observed for almost the whole region analyzed. Small amplifications of vibration amplitudes appear near the points where the forces are applied. The reduction effect for the vertical vibration component at a depth of $5 \mathrm{~m}$ is very similar to that for the ground surface (Figs. 5a, 6a). Looking at the horizontal components it can be observed that the attenuation effect is generally better for the points located near the ground surface (Figs. 5b, 6b and $5 \mathrm{c}, 6 \mathrm{c})$. The reduction of vibration amplitudes is always better for the points on the right side of the applied forces. The mitigation effect for the points located deeper below the ground surface is presented in Fig. 7, in the middle of the region considered, $y=$ $35 \mathrm{~m}$. The reduction effect for the vertical component $w$ is similar for different depths (Fig. 7a). For horizontal components $u$ and $v$ the reduction of vibration is worsened near the boundary surface between the first and the second layer (Fig. 7b, c). When a wave
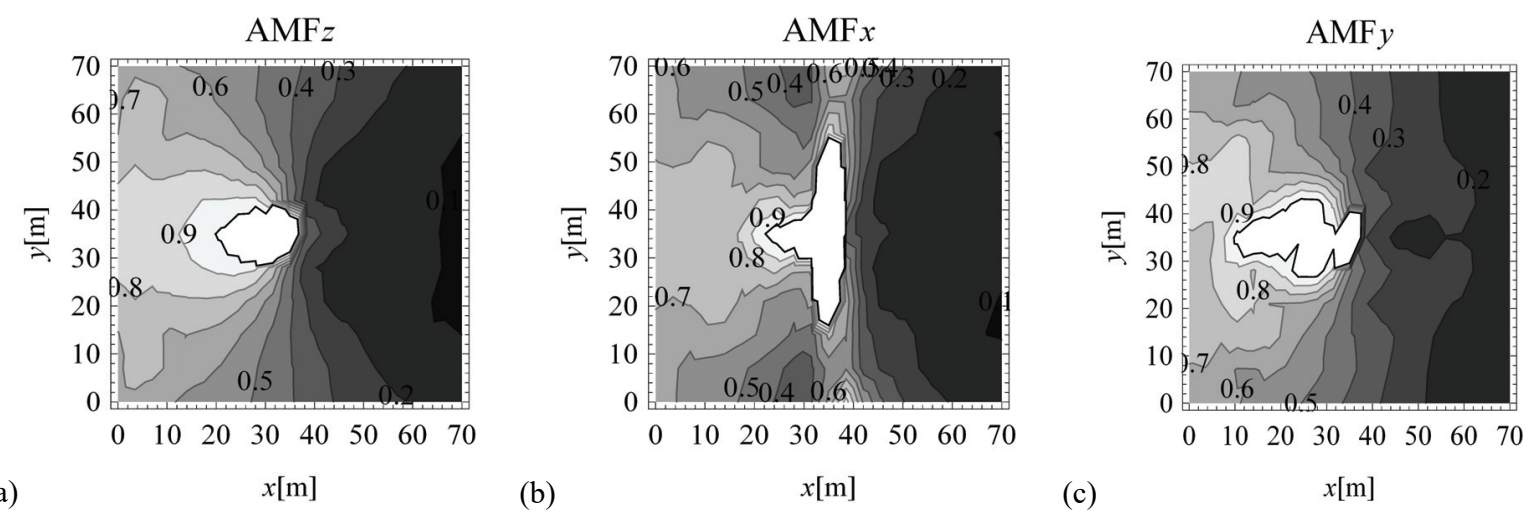

Fig. 5. AMF for the analyzed domain with the three displacement components for the points located on the ground surface, $r=2 \mathrm{~m}$; plot range: $0<\mathrm{AMF}<1$;

(a) displacement component in the $z$-direction, (b) displacement component in the $x$-direction,

(c) displacement component in the $y$-direction
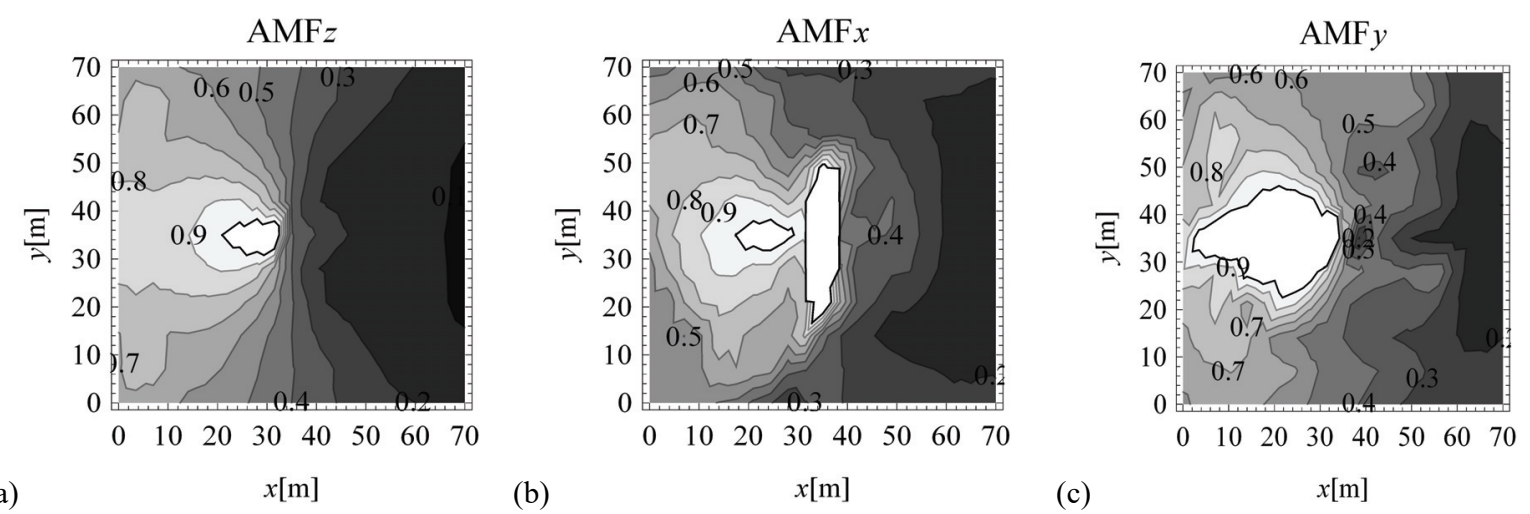

Fig. 6. AMF for the analyzed domain with the three displacement components for the points located $5 \mathrm{~m}$. below the ground surface, $r=2 \mathrm{~m}$; plot range: $0<\mathrm{AMF}<1$; (a) displacement component in the $z$-direction, (b) displacement component in the $x$-direction, (c) displacement component in the $y$-direction 


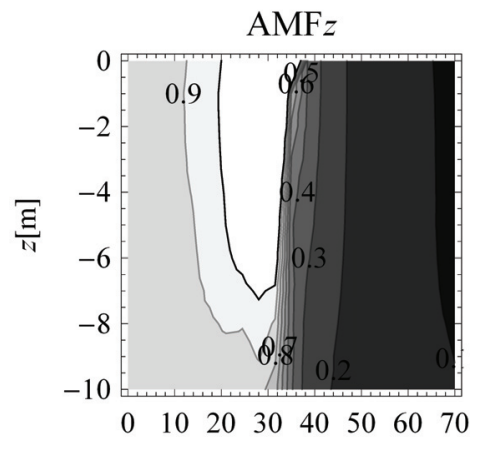

(a)

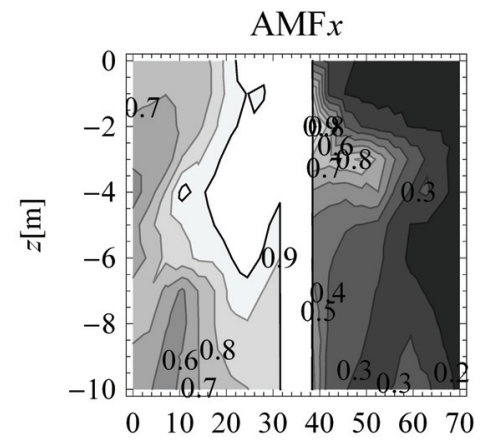

(b)

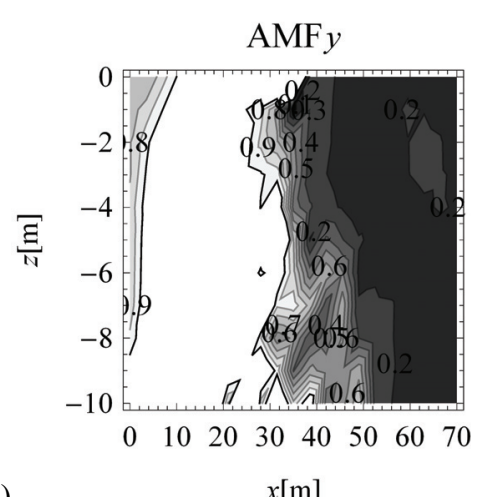

(c)

$x[\mathrm{~m}]$

Fig. 7. AMF with the three displacement components, for the points located below the ground surface, for $y=35 \mathrm{~m}, r=2 \mathrm{~m}$; plot range: $0<\mathrm{AMF}<1$; (a) displacement component in the $z$-direction, (b) displacement component in the $x$-direction, (c) displacement component in the $y$-direction
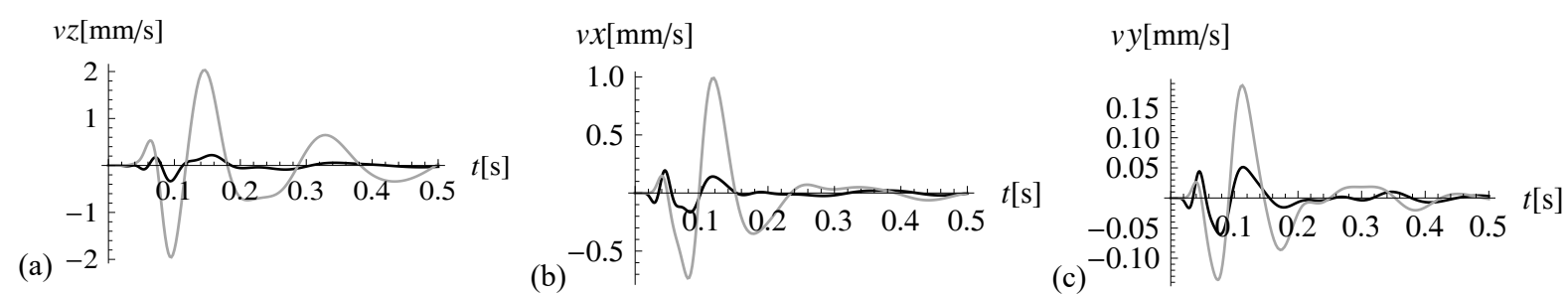

Fig. 8. Velocity components in the time domain at point 1 for $r=2 \mathrm{~m}$; black line: the additional generator is used; gray line: the additional generator is removed; (a) velocity component in the $z$-direction,

(b) velocity component in the $x$-direction, (c) velocity component in the $y$-direction

Table 1. AMF for the points 1-2 located on the foundation level and 3-6 located on the highest floor as in Fig. 1, for $r=2 \mathrm{~m}$

\begin{tabular}{|c|c|c|c|c|c|c|}
\hline AMF & Point 1 & Point 2 & Point 3 & Point 4 & Point 5 & Point 6 \\
\hline$z$-direction & 0.166 & 0.107 & 0.158 & 0.191 & 0.166 & 0.234 \\
\hline$x$-direction & 0.196 & 0.146 & 0.018 & 0.018 & 0.017 & 0.018 \\
\hline$y$-direction & 0.323 & 0.249 & 0.012 & 0.020 & 0.014 & 0.172 \\
\hline
\end{tabular}

impinges this boundary surface part of the energy is reflected and also refracted, new body waves appear. These new effects disturb the additional generator's efficiency. The disturbing effect is less visible at shallow depths because of the geometrical damping effect.

According to different European Standards for vibration control (DIN 4150-3, 1999 [9]; BS 5228-4, 1992 [2]; BS 7385-2, 1993 [3]) vibration monitoring should include velocity measurements at the foundation level and on structure, where the amplification of vibration can appear, as a result of the resonance effect. In Fig. 8 and Table 1, the reduction effect for a threestory building is presented. Velocity components in three directions $x, y, z$ are presented in time domain for point 1, located at the foundation level, as in Fig. 1. It can be observed that the significant reduction effect was achieved for all displacement components. The AMF values for points 1 and 2 are summarized in
Table 1, where also AMF values for points 3-6, located on the highest floor are presented (as in Fig. 1). The attenuation effect can be observed for all analyzed points, however exceptionally good results are obtained for horizontal vibrations on floors where the reduction effect greater than $98 \%$ was achieved (Table 1).

\subsection{THE SECOND LAYER'S DYNAMICAL PROPERTIES ARE BETTER}

In this section, similar analyses as in Section 3.1 are presented, but for the different soil conditions. In the case presented the second layer's dynamic properties are better, compared to the first one $G_{x z, 2}=2 G_{x z, 1}$. The layer's location and dynamic properties of the third and fourth layers stay the same as in the previous analyses (Fig. 1). It was verified that at such a depth changes in soil characteristics do not influence the 


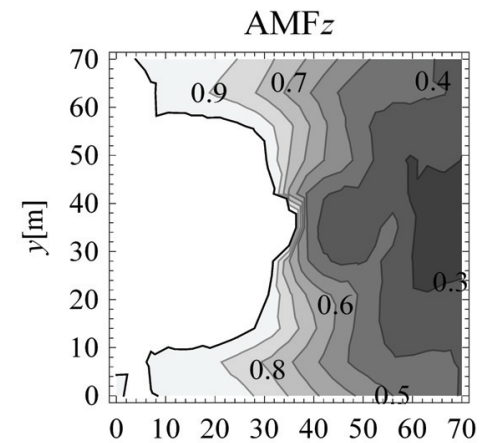

(a) $x[\mathrm{~m}]$

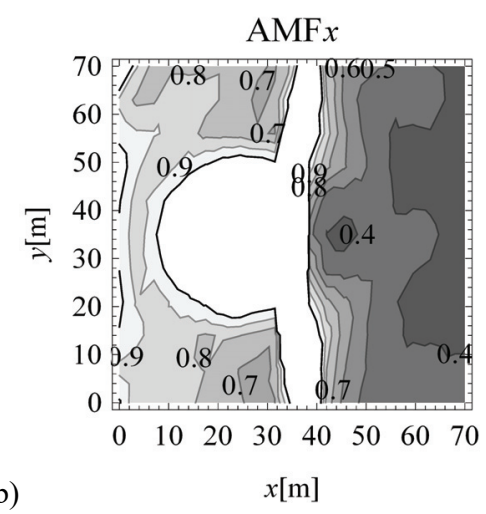

(b)

Fig. 9. AMF for the analyzed domain with the three displacement components

for the points located on the ground surface, $r=2 \mathrm{~m}$; plot range: $0<\mathrm{AMF}<1$; (a) displacement component in the $z$-direction, (b) displacement component in the $x$-direction,

(c) displacement component in the $y$-direction
$\mathrm{AMF} z$

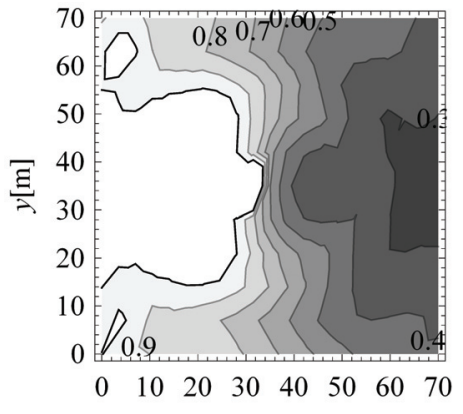

(a)

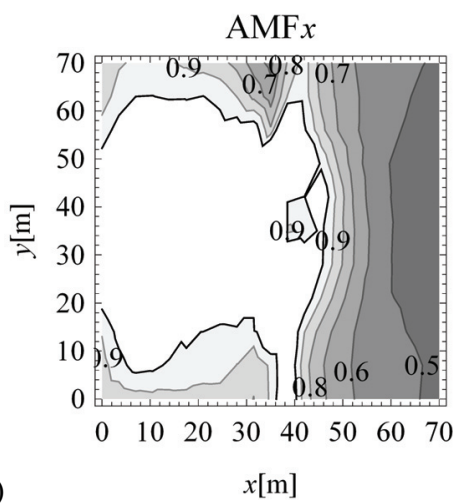

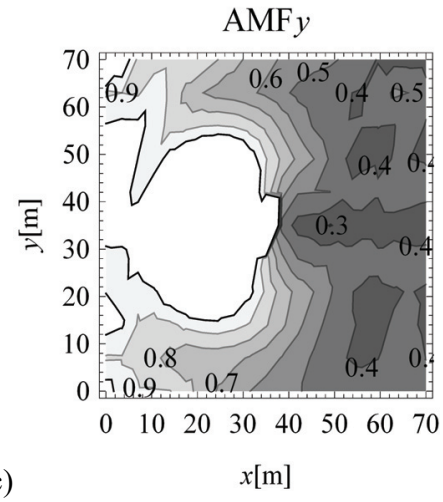

(b)

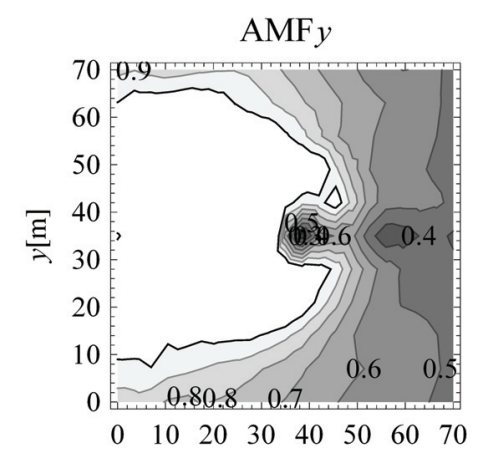

(c)

$x[\mathrm{~m}]$

Fig. 10. AMF for the analyzed domain with the three displacement components for the points located $5 \mathrm{~m}$ below the ground surface, $r=2 \mathrm{~m}$; plot range: $0<\mathrm{AMF}<1$;

(a) displacement component in the $z$-direction, (b) displacement component in the $x$-direction,

(c) displacement component in the $y$-direction

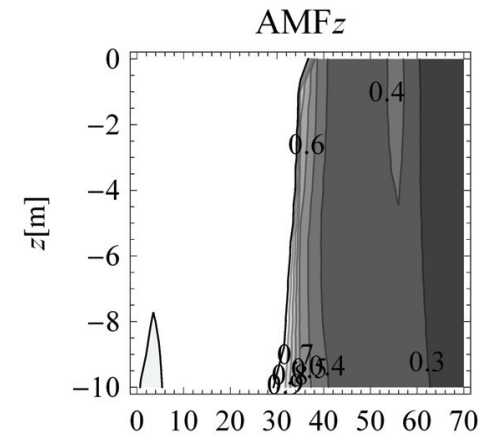

(a)

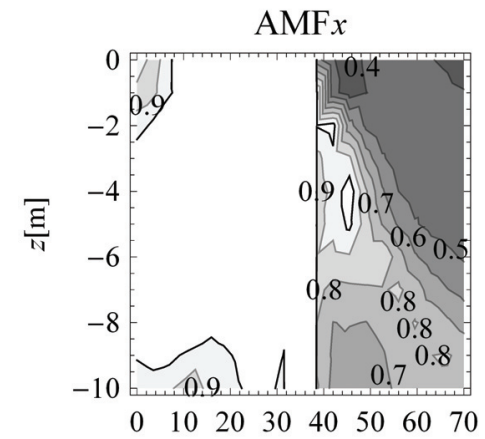

(b)

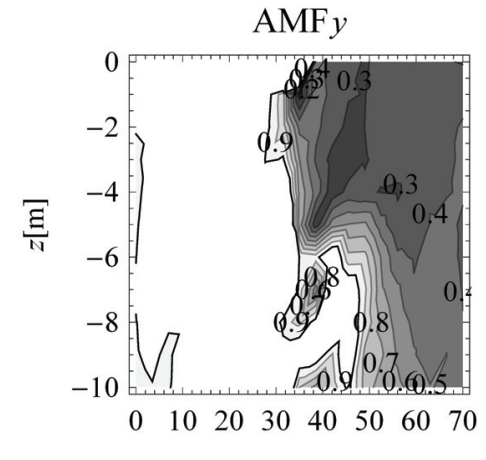

(c)

$x[\mathrm{~m}]$

Fig. 11. AMF with the three displacement components, for the points located below the ground surface, for $y=35 \mathrm{~m}, r=2 \mathrm{~m}$; plot range: $0<\mathrm{AMF}<1$; (a) displacement component in the $z$-direction, (b) displacement component in the $x$-direction, (c) displacement component in the $y$-direction

Table 2. AMF for the points 1-2 located on the foundation level and 3-6 located on the highest floor as in Fig. 1, for $r=2 \mathrm{~m}$

\begin{tabular}{|c|c|c|c|c|c|c|}
\hline AMF & Point 1 & Point 2 & Point 3 & Point 4 & Point 5 & Point 6 \\
\hline$z$-direction & 0.418 & 0.224 & 0.226 & 0.253 & 0.343 & 0.554 \\
\hline$x$-direction & 0.411 & 0.453 & 0.023 & 0.024 & 0.018 & 0.021 \\
\hline$y$-direction & 0.532 & 0.677 & 0.009 & 0.012 & 0.038 & 0.463 \\
\hline
\end{tabular}


amplitudes of the Rayleigh wave. Compared to the results obtained in Section 3.1 it can be observed that the reduction effect is worsened in the case of a weaker layer located at a shallow depth (Figs. 5 and 9, Figs. 6 and 10, Figs. 7 and 11). The regions of attenuated vibration amplitudes are smaller (Figs. 9-11). This corresponds with the results obtained in (Herbut [11]), where more extended analyses of soil conditions were presented. However, it should be emphasized that in each analyzed case the reduction effect on the right side of the region considered is observed. Similarly to the previous analyses (Section 3.1) the reduction effect for a vertical vibration component is similar at different depths (Fig. 11a). For horizontal components the reduction effect is generally worsened for deeper located points (Fig. 11 b, c). In Table 2, the results in the form of AMF are presented for the points located at the foundation level $(80 \mathrm{~cm}$ below the ground surface - points 1 and 2) and for the points located on the highest floor, as in Fig. 1 (points 3-6). For each analyzed point the reduction effect was achieved. However, the best results are obtained for horizontal vibration components on the highest floor.

\section{DISCUSSION AND CONCLUSIONS}

In this paper, the idea of an additional generator is presented and verified in the case of an impact load for two different soil conditions. The results in the form of a non-dimensional amplitude reduction factor are presented for the ground surface. The additional generator's efficiency for points located deeper below the ground surface is also verified. For all analyzed cases the reduction effect was achieved on the right side of the region considered. However, the best results were obtained when the stronger layer was located at the ground surface. For opposite layers location the mitigation effect was less significant. According to the present research, the idea proposed can be effective for structures with deep foundation location or underground structures. However, the reduction effect for horizontal displacement components was more visible for the points located near the ground surface than the ones below it.

Summarizing, the main advantages of the present approach are its simplicity and fast applicability. It does not require special work, such as installation of expensive devices on the structure or building an obstacle in the ground. That makes the solution relatively inexpensive and quick to implement. In my opinion, in the case of large value of Rayleigh wave- length when the application of trenches is not effective, an additional generator could be the most efficient approach to prevent large vibration amplitudes. The solution can be applied when large vibration amplitudes appear occasionally, i.e., in geotechnical works, when the installation of dampers in the structure is not reasonable. Additional generator can be used when the excitation force is applied directly to the ground surface, resulting in a dominant Rayleigh wave. The main disadvantage of the device presented is that it adds energy to the system, so it can increase vibration amplitudes if used incorrectly. This is the general weak point of all active approaches. Thus, vibration amplitudes must be monitored in buildings in the vicinity of an additional generator used, as is typical in geotechnical works (Rybak and Pieczyńska-Kozłowska [18]).

\section{ACKNOWLEDGMENTS}

The results of numerical calculations presented in this paper have been obtained with the support of the WCSS Computer Centre, within the frame of Grant No. 437.

\section{REFERENCES}

[1] Alzawi A., Hesham E., NAGgar M., Full scale experimental study on vibration scattering using open and in-filled (GeoFoam) wave barriers, Soil Dynamics and Earthquake Engineering, 2011, 31, 306-317.

[2] BS 5228-4:1992. British standard. Noise control on construction and open sites. Part 4: Code of practice of noise and vibration control applicable to piling operation.

[3] BS 7385-2:1993. Evaluation and measurement for vibration in buildings. Part 2: Guide to damage levels from ground borne vibration.

[4] CARCIONE J.M., Wave fields in real media: wave propagation in anisotropic, an elastic and porous media, Elsevier Science, Ltd., Amsterdam 2001.

[5] Chen X., Birk C., Song C., Numerical modelling of wave propagation in anisotropic soil using a displacement unitimpulse-response-based formulation of the scaled boundary finite element method, Soil Dynamics and Earthquake Engineering, 2014, 65, 243-255.

[6] Chu S.Y., Soong T.T., Reinhorn A.M., Active, hybrid and semi-active structural control: a design and implementation handbook, Chichester Wiley, 2005.

[7] Coulier P., Cuellar V., Degrande G., Lombaert G., Experimental and numerical evaluation of the effectiveness of a stiff wave barrier in the soil, Soil Dynamics and Earthquake Engineering, 2015, 77, 238-253.

[8] Dijckmans A., Ekblad A., Smekal A., Degrande G., LOMBAERT G., Efficacy of a sheet pile wall as a wave barrier for railway induced ground vibration, Soil Dynamics and Earthquake Engineering, 2016, 84, 55-69.

[9] DIN 4150-3:1999-02. Structural vibration Part 3: Effects of vibration on structures. 
[10] GaO G.Y., Li Z.Y., QIU C., YuE Z.Q., Three-dimensional analysis of rows of piles as passive barriers for ground vibration isolation, Soil Dynamics and Earthquake Engineering, 2006, 26, 1015-1027.

[11] HeRBUt A., A study of the reduction of ground vibrations by an active generator, Soil Dynamics and Earthquake Engineering, 2016, 88, 328-344.

[12] Kattis S.E., Polyzos D., Beskos D.E., Modelling of pile wave barriers by effective trenches and their screening effectiveness, Soil Dynamics and Earthquake Engineering, 1999, $18,1-10$.

[13] Leung K.L., Beskos D.E., Vardoulakis I.G., Vibration isolation using open or filled trenches Part 3: 2-D nonhomogeneous soil, Computational Mechanics, 1990, 7, 137-148.

[14] Lysmer J., Kuhlemeyer R.L., Finite dynamic model for infinite media, Journal of Engineering Mechanics Division, 1969, 95, 859-877.

[15] Mabsout M.E., Tassoulas S.L., A finite element model for the simulation of pile driving, Numerical Methods in Engineering, 1994, 37, 257-278.

[16] Pakos W., Wójcicki Z., Grosel J., Majcher K., SAWicki W., Experimental research of cable tension tuning of a scaled model of cable stayed bridge, Archives of Civil and Mechanical Engineering, 2016, 16(1), 41-52.
[17] Persson P., Persson K., SAndberg G., Reduction in ground vibrations by using shaped landscapes, Soil Dynamics and Earthquake Engineering, 2014, 60, 31-43.

[18] Rybak J., PieczyŃSKa-KozŁowska J.M., Vibration monitoring as a tool for a calibration of geotechnical technologies, 14th International Multidisciplinary Scientific GeoConference SGEM 2014: GeoConference on science and technologies in geology, exploration and mining : conference proceedings, Albena, Bulgaria, 17-26 June, 2014. Vol. 2, Hydrogeology, engineering geology and geotechnics. Sofia : STEF92 Technology, cop. 2014. 1043-1050.

[19] Turan A., Hafez D., El Naggar M.H., The performance of inclined secant micro-pile walls as active vibration barriers, Soil Dynamics and Earthquake Engineering, 2013, 55, 225-232.

[20] Ulgen D., Toygar O., Screening effectiveness of open and in-filled wave barriers: A full-scale experimental study, Construction and Building Materials, 2015, 86, 12-20.

[21] Verruijt A., An introduction to soil dynamics, Springer Verlag, Netherlands 2010.

[22] Woods R.D., Dynamics effects of pile installations on adjacent structures, National Academy Press, Washington D.C., 1997.

[23] Wrana B., Soil Dynamics. Computation models, Wydawnictwo Politechniki Krakowskiej, Kraków, 2016. 